\title{
关于卤素课堂教学的几点体会
}

朱国贤”, 潘荣楷

岭南师范学院化学化工学院, 广东 湛江 524048

摘要: 根据卤素的知识特点, 从讲述卤素发现史润物无声地开展思政教育, 运用化学基础理论渗透元素化学教学过程, 应用网络资源构建 “线上+线下” 混合模式教学, 结合实际应用巧讲卤素及其化合物, 指导学生学会归纳总结等方面进 行课堂教学, 能有效地激励学生学习科学家崇高的科学品质, 激发学生学习积极性, 提高课堂教学质量并提升学生的自 主学习能力。

关键词: 卤族; 教学改革; 思政教育; 元素化学

中图分类号: G64; O6

\section{Learning Experiences with Classroom Teaching of Halogens}

\section{Guoxian Zhu *, Rongkai Pan}

School of Chemistry and Chemical Engineering, Lingnan Normal University, Zhanjiang 524048, Guangdong Province, China.

\begin{abstract}
Based on the characteristics of halogens, classroom teaching is carried out by developing ideological and political educational content containing the history of the discovery of the halogen, permeating the teaching process of elemental chemistry with basic theoretical chemistry, constructing an "online + offline" mixed mode of teaching through the application of network resources, explaining halogens and their compounds with practical applications, guiding students to learn to induce and summarize the learned content, etc., thereby effectively motivating students to learn the scientific character of scientists, stimulating students' enthusiasm for learning, improving the quality of classroom teaching, and enhancing the self-learning ability of students.
\end{abstract}

Key Words: Halogen; Teaching reform; Ideological and political education; Elements chemistry

元素化学在日常生活和社会发展中有着极为广泛的用途, 常应用于能源、材料、医药、冶金、 环境等方面。元素化学是无机化学的主体部分, 主要讲述周期系中各主族、副族常见元素及其化合 物的存在、制备、结构、性质及其用途等有关知识 ${ }^{[1]}$, 是学生完成无机化学课程学习必不可少的重要 环节。由于元素化学的内容繁多琐碎, 知识点分散, 系统性差 ${ }^{[2]}$, 学生常感到 “一听就懂、一看就 烦、一放就忘、一用就乱” ; 教师则是花费大量时间和精力备课及上课却未能达到预期的教学效果, 因此, 元素化学的课堂教学常常出现学生 “难学”, 老师 “难教” 的困境。

如何精心设计课堂教学内容吸引学生的注意力? 如何有效运用教学方法和教学手段激发学生学 习兴趣? 如何有效地提高课堂教学质量? 如何能够在专业基础课教学中实现创新人才培养的目 标? 这一系列问题都是授课教师认真思考和亟需解决的重要课题。本文以 “卤素” 为例, 结合多年 大一的教学实践, 谈谈对卤素课堂教学的体会。

收稿: 2021-07-31; 录用: 2021-09-10; 网络发表: 2021-11-04 


\section{1 课堂教学设计思路}

卤素包括氟 $(\mathrm{F}) 、$ 氯 $(\mathrm{Cl})$ 、溴 $(\mathrm{Br})$ 、碘 $(\mathrm{I})$ 、砹 $(\mathrm{At}) 、$ 石田 $(\mathrm{Ts})$ 六种元素(常见前四种元素), 属于周期 系中第VIIA族元素, 位于元素周期表中的 $p$ 区。卤素是衔接中学化学与大学化学的第一族元素, 尽管 学生在中学阶段已经接触到卤素的知识, 但大学化学在知识的深度和广度上较中学化学都有较大的 提升, 而且大学化学更注重知识的内涵及外延, 注重了解事物本质, 注重让学生领会产生某现象的 原因, 理解各种公式及结论的缘由等。因此, 认清中学与大学化学的区别, 有助于授课教师合理地 设计课程教学内容和教学进度, 改进教学方法, 做到有的放矢开展教学, 使学生能够顺利完成从中 学到大学的过渡, 为今后的学习打下牢固的基础。

化学专业的人才培养目标是坚持学校 “师范性、教学型, 地方性、应用型” 的办学定位, 培养 德、智、体、美、劳全面发展, 掌握化学基本理论、基础知识和基本技能, 负责任、强能力、善创 新的优质应用型人才。根据无机化学课程标准, 编制卤素的教学要求: (1) 了解卤素的通性; (2) 掌 握氟的特殊性; (3) 掌握用元素电势图并用以判断卤素及其化合物的氧化还原性以及它们之间的相 互转化关系; (4) 掌握卤素单质结构、性质、制备和用途; (5) 掌握卤素氢化物、含氧酸及其盐的结 构、性质、制备和用途。为了更好地提高课堂教学效果, 更有效地达成教学目标, 元素化学课堂教 学内容应遵循以下原则 ${ }^{[3]}$ : 一是以基础理论作指导, 将基础理论的内容贯穿于元素化学的讲解中, 阐明单质及其化合物的物理与化学性质; 二是应用物质的性质决定其用途, 物质的用途体现其性质 的辨证关系, 通过介绍日常生活和生产相关的事例, 培养学生学习兴趣和系统掌握元素和化合物的 知识。以下具体谈谈 “卤素” 的教学内容组织与实践。

\section{2 课堂教学的内容组织与实践}

\section{1 结合思政教育, 上好第一堂课}

俗语说: “万事开头难” 。卤素是大一学生学习元素化学时首先学习的第一族元素, 具有承上 启下作用, 上好第一节课至关重要。课堂可运用元素发现史导入新课, 并向学生说明任何一个科学 发现或发明, 无不凝聚着化学家的不畏艰险、顽强探索, 勇攀高峰, 甚至为科学献身的科学精神。 授课时, 可利用3-4分钟讲述单质氟(氟气)的发现过程。在化学元素发现史上, 持续时间最长的、参 加人数最多的、危险最大的, 莫过于单质氟的制取了。自 1774 年瑞典化学家舍勒在研究硫酸与萤石 的反应时发现 $\mathrm{HF}^{[4]}$, 到1886年6月法国化学家亨利·莫瓦桑第一次制得氟的气态单质, 历时 110 多年之 久, 在漫漫征程中, 英国化学家汉弗里·戴维, 爱尔兰的乔治·诺克斯和托马斯·诺克斯两兄弟因氟而 严重中毒, 比利时化学家鲁耶特和法国的尼克雷不幸殉难。莫瓦桑总结了前人的经验教训, 研究了 几乎全部有关氟及化合物的著作。尽管当时实验室条件极差, 他也 3 次中毒, 但他不灰心、不气馁、 不放弃, 百折不挠, 反复尝试, 终于用电解法从加入氟化钾的氟化氢液体中制得单质氟(氟气) ${ }^{[5]}$ 。 1892年和1893年, 他又连续先后发明了高温电炉一一莫氏炉和人造金刚石。由于莫瓦桑这些震惊世 界的杰出成就, 1906年, 他获得了科学界的最高荣誉——诺贝尔化学奖。遗憾的是, 1907年2月, 莫 瓦桑不足 55 岁便与世长辞。同时教师也要指出氟单质的发现过程, 是无数化学家用他们的血泪甚至 生命积聚的过程, 是化学史上极其悲壮的一段历程。这段悲壮历程展现了人类对未知世界的渴望, 体现了人类文明最可贵的精神!

通过讲述氟单质的 “小故事” , 可激发学生对化学的求知欲望和探索精神, 又能悄然无声地开 展思想品德教育, 引导学生学习科学家追求真理、坚毅执着、严谨求实、献身科学的高尚品德。

课后, 要求学生查阅资料, 自学氯、溴、碘元素的发现史, 写一篇题目为 “卤素发现史给我的 启示” 的文章, 以此培养学生不怕挫折、勇于向困难挑战的勇气, 激励学生勤奋学习, 刻苦钻研, 锐意进取，为实现中华民族的伟大复兴贡献自己的一份力量。

\section{2 将基础理论渗透元素化学教学中}

化学基础理论与元素化学之间存在着相互作用、相辅相成的辩证关系, 基础理论对元素化学教 
学具有指导作用, 而学好元素化学又会加深对基础理论的理解和应用。

\subsection{1 以物质结构理论为指导}

在本章教学中, 运用原子和分子结构理论来解释物质的结构, 阐明物质的性质, 让学生理解结 构与性质之间的内在联系, 可使罗列材料、平铺直叙的教学转变为具有逻辑性的、推理性的教学, 帮助学生理解本族元素及化合物的性质 ${ }^{[6]}$ 。

例如在讲述卤素的通性时, 可运用原子结构和元素周期律等知识说明卤素的性质。由于卤素原 子的价电子构型为 $n s^{2} n p^{5}$, 都有获取一个电子以达到 8 电子稳定结构的强烈倾向, 单个原子的化学性 质很活泼, 卤素单质都是双原子分子, 在自然界中难以游离状态存在。从氟到碘, 随着原子序数不 断增加, 原子半径的不断增大, 卤素分子之间的色散力逐渐增大, 颜色由浅变深, 状态由气态、液 态到固态, 熔沸点逐渐升高, 密度逐渐增大, 溶解性逐渐减小等, 这是结构决定性质的一个典型例 子。

又如在讲述卤素含氧酸的强度时, 其水溶液的酸性强弱可从结构上得到解释。当中心原子 R 的半 径较小, 电负性较大, 氧化数较高时, $\mathrm{R}$ 原子极化力较强, $\mathrm{R}$ 原子吸引羟基氧原子的电子的能力较强, 能有效地降低氧原子上的电子密度, 使 $\mathrm{O}-\mathrm{H}$ 键变弱, 有利于释放出质子, 而表现出较强的酸性, 不 难推出同一族同种类型的含氧酸, 其酸性自上至下依次减弱, 如: $\mathrm{HClO}_{4}>\mathrm{HBrO}_{4}>\mathrm{H}_{5} \mathrm{IO}_{6}$; 同理可 以推断: 对于同一元素不同氧化态的含氧酸, 则高氧化态含氧酸的酸性较强, 低氧化态含氧酸的酸 性较弱, 如: $\mathrm{HClO}_{4}>\mathrm{HClO}_{3}>\mathrm{HClO}_{2}>\mathrm{HClO}$ 。

因此, 沿着结构决定性质这一主线开展教学, 可有助于学生加深对本族元素及其化合物的性质 的理解, 又可加深对其性质的记忆, 同时培养了学生运用理论知识分析实际问题的能力, 起到事半 功倍的教学效果。

\subsection{2 以元素电势图为指导}

元素电势图是学习元素化学时非常有用的工具, 除IA、IIA外, 元素化学中每一族元素均有介绍 该族元素的电势图。元素电势图是指同一元素的各种氧化态之间, 各个电对的标准电极电势变化的 关系图。如氯元素在酸及碱性介质的电势图如图1所示。

$$
\begin{aligned}
& \varphi_{A}{ }^{\ominus} / \mathrm{V}: \\
& \mathrm{ClO}_{4}{ }^{-} \stackrel{1.19}{\mathrm{ClO}_{3}}{ }^{-} \stackrel{1.21}{=} \mathrm{HClO}_{2} \stackrel{1.64}{\mathrm{HClO}} \stackrel{1.63}{=} 1 / 2 \mathrm{Cl}_{2} \stackrel{1.358}{=} \mathrm{Cl}^{-} \\
& \varphi_{B}{ }^{\Theta} / \mathrm{V}: \\
& \mathrm{ClO}_{4}{ }^{-} \stackrel{0.36}{-} \mathrm{ClO}_{3}{ }^{-} \stackrel{0.35}{ }-\mathrm{ClO}_{2}{ }^{-} \stackrel{0.566}{ } \quad \mathrm{ClO}^{-} \stackrel{0.40}{\longrightarrow} 1 / 2 \mathrm{Cl}_{2} \stackrel{1.358}{\longrightarrow} \mathrm{Cl}^{-}
\end{aligned}
$$

图1 氯的电势图(本文所用数据, 均来自文献 ${ }^{11}$ )

应用电势图可以了解元素及化合物的许多基本性质, 例如元素的常见氧化态(如图1中 $\mathrm{Cl}$ 的氧化 态为 $+7,+5,+3,+1,0,-1$ )及相应的物质(如在酸性介质存在: $\mathrm{ClO}_{4}^{-}, \mathrm{ClO}_{3}^{-}, \mathrm{HClO}_{2}, \mathrm{HClO}, \mathrm{Cl}_{2}$, $\mathrm{Cl}^{-}$), 元素不同氧化态的氧化还原性强弱, 介质酸碱性对氧化还原性的影响(如图1中电对在酸性介质 的氧化性比相应的电对在碱性介质中强), 以及判断中间氧化态的物质能否发生歧化反应(如图1在酸 性介质中 $\mathrm{ClO}_{3}^{-}, \mathrm{HClO}_{2}$ 发生歧化反应; 在碱性介质中 $\mathrm{ClO}_{2}^{-}, \mathrm{Cl}_{2}$ 发生歧化反应)等。另外, 应用元素电 势图, 可以判断氧化还原反应的产物, 如讲述 $\mathrm{I}_{2}$ 与碱反应时, 向学生提问: 根据碘的元素电势图(见 图2), 判断单质碘在标态下(以下讨论均在标态), 在碱性溶液中是否发生歧化反应? 歧化产物是什 么?

从图2可知: $\mathrm{I}_{2}$ 在碱性介质中可以自发发生歧化反应 $\left(\varphi_{\text {石 }}^{\ominus}>\varphi_{\text {左}}^{\ominus}\right.$, 歧化产物是 $\mathrm{IO}^{-}$和 $\mathrm{I}^{-}$, 即: $\mathrm{I}_{2}+$ $2 \mathrm{OH}^{-}=\mathrm{I}^{-}+\mathrm{IO}^{-}+\mathrm{H}_{2} \mathrm{O}$ 。这时可进一步提问: $\mathrm{IO}^{-}$是不是歧化反应的最终产物? 让学生思考片刻后, 
老师作出解释： $\mathrm{IO}^{-}$不是歧化反应的最终产物。因为生成的 $\mathrm{IO}^{-}$还将进一步发生歧化反应, 并存在两 种反应方式: 第一种是歧化生成 $\mathrm{IO}_{3}^{-}$和 $\mathrm{I}_{2}$, 这时, $E^{\Theta}=\varphi_{\text {石 }}^{\ominus}-\varphi_{\text {左 }}^{\Theta}=0.445-0.145=0.300(\mathrm{~V})$; 另一种 是歧化生成 $\mathrm{IO}_{3}^{-}$和 $\mathrm{I}^{-}$, 这时, $E^{\Theta}=\varphi_{\text {石 }}^{\ominus}-\varphi_{\text {左 }}^{\ominus}=0.49-0.145=0.345(\mathrm{~V})$ 。一般来说, 不考虑动力学因 素, 氧化还原反应进行的次序是电动势较大的反应优先进行。因此, $\mathrm{IO}^{-}$将按第二种歧化方式优先进 行反应, 生成 $\mathrm{IO}_{3}^{-}$和 $\mathrm{I}^{-}$。即使 $\mathrm{IO}^{-}$可以同时进行第一种歧化方式反应生成 $\mathrm{IO}_{3}^{-}$和 $\mathrm{I}_{2}$, 则生成的 $\mathrm{I}_{2}$ 将继续歧 化生成 $\mathrm{IO}_{3}^{-}$和 $\mathrm{I}^{-}$, 因为 $\varphi^{\Theta}\left(\mathrm{IO}_{3}^{-} / \mathrm{I}_{2}\right)=(0.145 \times 4+0.455 \times 1) / 5=0.205(\mathrm{~V})$, 这时, $E^{\Theta}=\varphi_{\text {石 }}^{\Theta}-\varphi_{\text {左 }}^{\Theta}=0.535-$ $0.205=0.330(\mathrm{~V})$ 。若把上述电对按标准电极电势大小由低到高排序见表 1,1 号电对与 6 号电对构成 原电池的电动势数值最大, 该反应优先进行, 即: $3 \mathrm{I}_{2}+6 \mathrm{OH}^{-}=5 \mathrm{I}^{-}+\mathrm{IO}_{3}^{-}+3 \mathrm{H}_{2} \mathrm{O}$ 。实际上, 不论是 $\mathrm{I}_{2}$ 直接歧化生成 $\mathrm{IO}_{3}^{-}$和 $\mathrm{I}^{-}$, 还是先歧化生成 $\mathrm{IO}^{-}$和 $\mathrm{I}^{-}$, 在碱性溶液中歧化的最终产物都是 $\mathrm{I}^{-}$和 $\mathrm{IO}_{3}^{-}$。实验证 明, 单质碘即使在冷的碱溶液中也能迅速歧化生成 $\mathrm{IO}_{3}^{-}$和 $\mathrm{I}^{-}$。

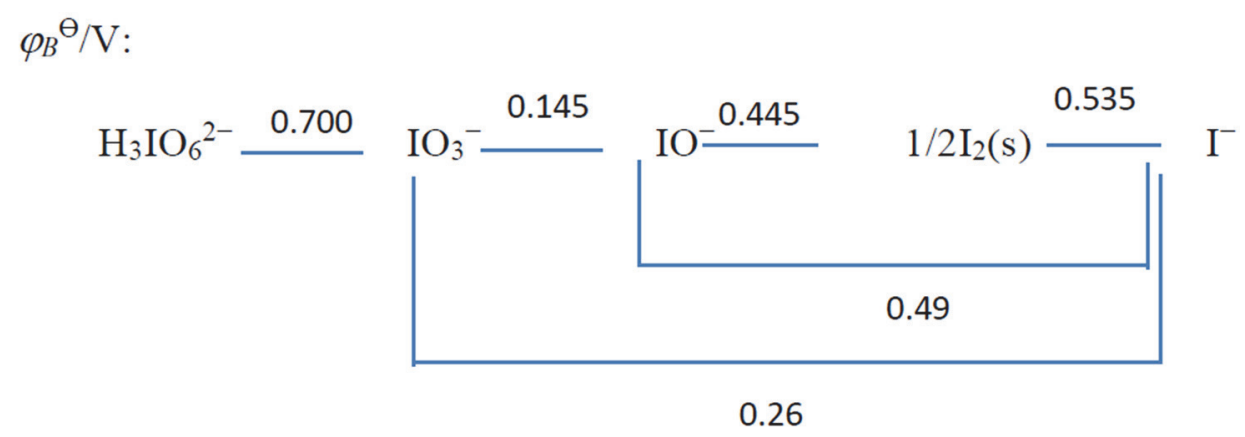

图2 碘的电势图

表1＼cjkstart标准电极电势由低到高排序表

\begin{tabular}{ccc}
\hline 序号 & 电对(氧化状态/还原状态) & $\varphi_{\mathrm{B}}^{\Theta} / \mathrm{V}$ \\
\hline 1 & $\mathrm{IO}_{3}^{-} / \mathrm{IO}^{-}$ & 0.145 \\
2 & $\mathrm{IO}_{3}^{-} / \mathrm{I}_{2}$ & 0.205 \\
3 & $\mathrm{IO}_{3}^{-} / \mathrm{I}^{-}$ & 0.26 \\
4 & $\mathrm{IO}^{-} / \mathrm{I}_{2}$ & 0.445 \\
5 & $\mathrm{IO}^{-} / \mathrm{I}^{-}$ & 0.49 \\
6 & $\mathrm{I}_{2} / \mathrm{I}^{-}$ & 0.535 \\
\hline
\end{tabular}

因此, 指导学生观察和分析元素电势图, 运用元素电势图进一步分析化学反应方程式, 有助于 学生了解化学反应的内在规律, 有助于学生记忆化学方程式。

\subsection{3 以热力学与动力学理论为指导}

化学热力学是从能量变化的角度研究化学反应的科学, 着眼于宏观性质的变化, 可以解决化学 反应进行的方向和限度等问题; 化学动力学研究化学反应快慢(即速率)和微观历程(或称机理), 以及 各种因素对反应速率的影响。两种理论紧密联系, 相得益彰, 若把两种理论应用到元素化学教学中, 可以合理地解释许多化学反应现象, 进一步认识化学反应的规律。

卤素单质 $\left(\mathrm{F}_{2}\right.$ 除外)与水反应是个典型的例子。卤素单质 $\left(\mathrm{F}_{2}\right.$ 除外 $)$ 与水发生可逆的歧化反应, 反应 方程式为: $\mathrm{X}_{2}+\mathrm{H}_{2} \mathrm{O} \rightleftharpoons \mathrm{HXO}+\mathrm{HX}(\mathrm{X}=\mathrm{Cl}, \mathrm{Br}, \mathrm{I})$ 。但反应程度较差, 且从氯、溴、碘逐步减弱, 详 见表2。往溶液加碱, 增加溶液的 $\mathrm{pH}$, 因 $\mathrm{OH}^{-}+\mathrm{H}^{+}=\mathrm{H}_{2} \mathrm{O}$, 降低了生成物的浓度, 平衡向右移动, 相 当于卤素在碱性溶液中发生歧化反应, 并存在以下两种反应方式:

$$
\mathrm{X}_{2}+2 \mathrm{OH}^{-} \rightarrow \mathrm{X}^{-}+\mathrm{OX}^{-}+\mathrm{H}_{2} \mathrm{O}
$$




$$
3 \mathrm{X}_{2}+6 \mathrm{OH}^{-} \rightarrow 5 \mathrm{X}^{-}+\mathrm{XO}_{3}^{-}+3 \mathrm{H}_{2} \mathrm{O}
$$

$\mathrm{X}_{2}$ 歧化反应的实际产物是由动力学因素一反应速率决定: $\mathrm{Cl}_{2}$ 在 $20^{\circ} \mathrm{C}$ 时, 反应(1)进行得很快, 在 $70^{\circ} \mathrm{C}$ 时, 反应 $(2)$ 才进行得很快, 因此, 常温下 $\mathrm{Cl}_{2}$ 与碱作用主要生成次氯酸盐。 $\mathrm{Br}_{2}$ 在 $20^{\circ} \mathrm{C}$ 时两个 反应都进行很快, 而在 $0^{\circ} \mathrm{C}$ 时反应(2)较缓慢, 因此只有在 $0^{\circ} \mathrm{C}$ 时才能得到次溴酸盐。 $\mathrm{I}_{2}$ 在 $0{ }^{\circ} \mathrm{C}$ 时反 应(2)也进行得很快, 所以 $\mathrm{I}_{2}$ 与碱反应只能得到碘酸盐。经过详细的分析和讲解, 让学生能深刻地理 解卤素 $\left(\mathrm{F}_{2}\right.$ 除外 $)$ 与水反应的规律。

表2 在 $298 \mathrm{~K}$ 下卤素饱和水溶液的平衡浓度

\begin{tabular}{ccccc}
\hline 卤素单质 & 溶解度 $/\left(\mathrm{mol} \cdot \mathrm{L}^{-1}\right)$ & $\mathrm{X}_{2}(\mathrm{aq})$ 浓度 $/\left(\mathrm{mol} \cdot \mathrm{L}^{-1}\right)$ & $\mathrm{HOX}$ 浓度 $/\left(\mathrm{mol} \cdot \mathrm{L}^{-1}\right)$ & $K^{\ominus}$ \\
\hline $\mathrm{Cl}_{2}$ & 0.0921 & 0.062 & 0.030 & $4.2 \times 10^{-4}$ \\
$\mathrm{Br}_{2}$ & 0.214 & 0.210 & $1.1 \times 10^{-3}$ & $7.2 \times 10^{-9}$ \\
$\mathrm{I}_{2}$ & 0.0013 & 0.0013 & $6.4 \times 10^{-6}$ & $2.0 \times 10^{-13}$ \\
\hline
\end{tabular}

事实上卤素与水反应是教学的重点、难点, 相当一部分学生从这部分内容开始, 感到元素化学 知识点繁多、内容琐碎, 尤其是众多的化学反应方程式, 学习时难以记忆, 产生庆学、怕学情绪。 然而, 将基础理论渗透元素化学教学中, 立足于物质结构理论, 结合化学热力学、化学动力学、元 素电势图等理论, 把基础理论与元素化学互相穿插、有机揉合, 从微观和宏观的角度深入细致地分 析物质的性质, 揭示元素及其化合物的内在联系及其变化规律, 这样, 可使元素化学庞杂零散的知 识显得有条不紊、井然有序, 有助于学生理解、记忆、掌握并巩固元素化学的知识。

\section{3 借助网络资源, 推动课堂教学模式改革}

随着互联网和移动通信技术日新月异的发展, 极大地改变了知识传播的方式, 学生不受时间和 空间的界限, 随时可有效地获取知识, 这无疑推动传统课堂教学模式发生深刻变革, 也对教师提出 了更高的要求。这需要高校教师深入钻研教材, 挖掘教材, 潜心备课, 把握教材重点及难点, 改进 教学模式和教学手段, 熟练掌握教学方法及信息技术的运用, 准确了解学生的学习情况等。课堂上, 教师要充分发挥对学生学习的引领和指导作用, 努力实现以学生为主体、教师为主导的有效课堂。

例如, 在讲授14.2卤素单质时, 课前按教学要求预先设计符合学生认知发展规律的教学思考题 (见表3)：1）给出卤素单质的活泼性次序? 为什么由氟至氯活泼性变化有一个突变? 2) 试解释为何 $\mathrm{I}_{2}$ 溶解在 $\mathrm{CCl}_{4}$ 中得到紫色溶液, 而 $\mathrm{I}_{2}$ 在乙醚中却是红棕色? 3) 试解释为何 $\mathrm{I}_{2}$ 难溶于水却易溶于 $\mathrm{KI}$ 中? 4) 讨论 $\mathrm{Cl}_{2}, \mathrm{Br}_{2}, \mathrm{I}_{2}$ 与 $\mathrm{NaOH}$ 溶液作用的产物及条件; 5) 电解制氟时, 为何不用 $\mathrm{KF}$ 的水溶液? 6) 用 反应方程式表示由氯化钾制备氧气及由海水制溴酸的过程; 7) 氯气长时间通入KI溶液中, 溶液颜色 由黄色变为近无色, 试用方程式表示?

这些问题要求学生课前通过慕课或精品视频公开课或资源共享等网络平台观看视频, 自主学习, 思考并回答这些问题, 把完成任务的情况通过师生交流平台(已建立的微信、QQ交流群或雨课堂等) 反馈给老师。老师对学生完成任务的具体情况进行收集、归纳、分析、整理, 了解学生学习中存在 的疑惑和问题, 研究教学方法和教学手段, 进而精心设计课堂教学的各个环节。课堂上, 教师以问 题为导向, 根据课程内容和学生学习情况, 灵活采用启发式、探究式、互动式等方法进行集中讲解, 讲清重点, 讲透难点; 对学习中存在的问题, 教师组织学生进行分组讨论、探讨、研究, 启发学生 开拓思路, 积极思考, 激发学生的潜能, 引导学生用已学的知识去分析问题、解答问题, 鼓励学生 踊跃发言, 指导学生寻找解决问题的方法, 从而让学生更好地全面掌握知识。

借助网络资源, 构建 “线上+线下” 教学过程, 采用 “设置问题 $\rightarrow$ 自主学习 $\rightarrow$ 答辩释疑 $\rightarrow$ 解惑提 升” 的混合式教学模式, 推动传统教学模式的改革, 能有效引导学生多读书、深思考、善提问、勤 实践, 把 “要我学” 变为 “我要学”, 大大提高学生课堂参与度, 提高学生对元素化学的学习热情 度, 提升学生的自主学习能力和独立思考能力, 有利于促进学生个性的发展, 培养学生的科学思 维, 开发学生的创新潜能, 充分体现学生的主体地位。 
大学 化学 Univ. Chem. 2022,37(6),2107126 (6 of 8)

表3 卤素课前部分学习思考题与简答* ${ }^{[1,7]}$

\begin{tabular}{|c|c|c|c|}
\hline 教学内容 & 教学要求 & 问题 & 简答 \\
\hline \multirow[t]{27}{*}{14.2 卤素单质 } & 掌握卤素单质结构、 & 1) 给出卤素单质的活泼性次序? & 1) 单质的活泼性次序为: $\mathrm{F}_{2}>>\mathrm{Cl}_{2}>\mathrm{Br}_{2}>\mathrm{I}_{2}$; \\
\hline & 性质、制备和用途 & 为什么由氟至氯活泼性变化有一 & 从 $\mathrm{F}_{2}$ 到 $\mathrm{Cl}_{2}$ 活泼性突变, 其原因归结为 $\mathrm{F}$ 原子和 $\mathrm{F}^{-}$离子的 \\
\hline & & 个突变? & 半径特别小 \\
\hline & & 2) 试解释为何 $\mathrm{I}_{2}$ 溶解在 $\mathrm{CCl}_{4}$ 中得 & 2) $\mathrm{CCl}_{4}$ 为非极性溶剂, $\mathrm{I}_{2}$ 溶在 $\mathrm{CCl}_{4}$ 中后仍为分子状态, \\
\hline & & 到紫色溶液, 而 $\mathrm{I}_{2}$ 在乙醚中却是红 & 显示出 $\mathrm{I}_{2}$ 单质在蒸气时的紫颜色; 而乙醚为极性溶剂, \\
\hline & & 棕色? & $\mathrm{I}_{2}$ 溶于乙梄时与溶剂间有溶剂合作用, 形成的溶剂合物 \\
\hline & & & 不再呈其单质蒸气时的紫色, 而是呈红棕色 \\
\hline & & 3) 试解释为何 $I_{2}$ 难溶于水却易溶 & 3) $I_{2}$ 以分子状态存在, 在水中歧化部分很少, 按相似相 \\
\hline & & 于KI中? & 溶的原则, 非极性的 $\mathrm{I}_{2}$ 在水中溶解度很小。但 $\mathrm{I}_{2}$ 在 $\mathrm{KI}$ 溶 \\
\hline & & & 液中与 $\mathrm{I}^{-}$相互作用生成 $\mathrm{I}_{3}$ 离子, $\mathrm{I}_{3}$ 离子在水中的溶解度 \\
\hline & & & 很大 \\
\hline & & 4) 讨论 $\mathrm{Cl}_{2}, \mathrm{Br}_{2}, \mathrm{I}_{2}$ 与 $\mathrm{NaOH}$ 溶液 & 4) 详见2.2.3以热力学与动力学理论为指导 \\
\hline & & 作用的产物及条件 & \\
\hline & & 5) 电解制氟时, 为何不用 $K F$ 的水 & 5) 因为电解 $K F$ 的水溶液, 生成的 $F_{2}$ 能与水发生剧烈反 \\
\hline & & 溶液？ & 应, 而得不到单质氟, 得到的是 $\mathrm{O}_{2}$, 所以不能用 $\mathrm{KF}$ 的水 \\
\hline & & & 溶液 \\
\hline & & & $2 \mathrm{~F}_{2}+2 \mathrm{H}_{2} \mathrm{O}=4 \mathrm{HF}+\mathrm{O}_{2}$ \\
\hline & & 6) 用反应方程式表示: 由氯化钾 & 6) (1) $2 \mathrm{KCl}+2 \mathrm{H}_{2} \mathrm{O}=\mathrm{Cl}_{2} \uparrow+2 \mathrm{KOH}+\mathrm{H}_{2} \uparrow$ (电解) \\
\hline & & 制备氧气和由海水制溴 & 电极不用隔膜, 加热后, $\mathrm{Cl}_{2}$ 歧化 \\
\hline & & & $3 \mathrm{Cl}_{2}+6 \mathrm{KOH}=5 \mathrm{KCl}+\mathrm{KClO}_{3}+3 \mathrm{H}_{2} \mathrm{O}$ \\
\hline & & & $2 \mathrm{KClO}_{3}=2 \mathrm{KCl}+3 \mathrm{O}_{2} \uparrow\left(\mathrm{MnO}_{2}\right.$ 作催化剂 $)$ \\
\hline & & & (2) $\mathrm{Cl}_{2}+2 \mathrm{Br}^{-}=2 \mathrm{Cl}^{-}+\mathrm{Br}_{2}$ \\
\hline & & & $3 \mathrm{Na}_{2} \mathrm{CO}_{3}+3 \mathrm{Br}_{2}=5 \mathrm{NaBr}+\mathrm{NaBrO}_{3}+3 \mathrm{CO}_{2}$ \\
\hline & & & $5 \mathrm{Br}^{-}+\mathrm{BrO}_{3}^{-}+6 \mathrm{H}^{+}=3 \mathrm{Br}_{2}+3 \mathrm{H}_{2} \mathrm{O}$ \\
\hline & & 7) 氯气长时间通入 $K I$ 溶液中, 溶 & 7) $\mathrm{Cl}_{2}+2 \mathrm{KI}=2 \mathrm{KCl}+\mathrm{I}_{2}$ \\
\hline & & 液颜色由黄色变为近无色, 试用 & $5 \mathrm{Cl}_{2}+\mathrm{I}_{2}+6 \mathrm{H}_{2} \mathrm{O}=2 \mathrm{HIO}_{3}+10 \mathrm{HCl}$ \\
\hline & & 方程式表示？ & • \\
\hline
\end{tabular}

*表3中的章节名称、顺序参照文献 ${ }^{[1]}$

\section{4 结合实际应用, 讲透卤素及其重要化合物}

卤素化学丰富多彩, 涉及面广, 能形成卤化物、卤素互化物、多卤化物, 含氧酸及其盐等多种 类型的化合物, 是教学的重点, 更是教学的难点, 尤其是出现大量体现化合物的各种性质的化学反 应方程式, 而且物质性质中有相当部分又无法用基础理论圆满解释, 这无疑给教学带来极大困难, 老师在教学过程中会产生迷惑的感觉, 学生在学习过程中因难以把握重点及记牢知识而产生畏难、 甚至有放弃的想法。显然, 如何提高学生的学习兴趣是老师首要思考的问题, 正如教育家布鲁纳说: “学习的最好刺激, 乃是对所学材料的兴趣。” 因此, 在教学中, 通过介绍该化合物在日常生活与 生产中的作用, 增加课堂教学的知识性、趣味性, 活跃课堂气氛, 这样有助于激发学生学习元素化 学知识的主动性和自觉性, 让学生学会学习, 学会思考, 学会分析问题和解决问题。

例如, 讲授氯酸盐( 以氯酸钾为代表)时, 先讲述氯酸钾用途, 它可用于炸药、烟花、火柴、医药 等。这些应用说明氯酸钾是强氧化剂, 接着讲授氯酸钾的氧化性, 如有催化剂如 $\mathrm{MnO}_{2}$ 等存在, 在较 低温度下就能分解且强烈放出氧气: $2 \mathrm{KClO}_{3}=2 \mathrm{KCl}+3 \mathrm{O}_{2}\left(\mathrm{MnO}_{2}\right.$ 作催化剂 $)$; 在酸性溶液中有强氧化 
作用, 可把碘氧化成碘酸: $2 \mathrm{ClO}_{3}^{-}+2 \mathrm{H}^{+}+\mathrm{I}_{2}=2 \mathrm{HIO}_{3}+\mathrm{Cl}_{2} \uparrow$; 与碳、磷及有机物或可燃物混合受到 撞击时, 易发生燃烧和爆炸等。由此还可推断氯酸钾的储存方式: 不宜在日光下长时间曝晒, 并防 止与有机物和其他易氧化物接触, 密封阴凉保存; 由于氯酸钾是强氧化剂, 制备时常采用电解法。 又如含氟牙膏有效预防龉齿, 氯气用作消毒剂、漂白剂, 医疗上用 $0.9 \%$ 的氯化钠溶液做生理盐水, 澳化银可用于制胶卷和相纸, 碘化银可用于人工降雨, 食用碘盐可以预防甲状腺肿大等, 这些耳熟 能详的事例均是很好的教学素材。

沿着 “性质决定用途, 用途体现性质” 的思路开展课堂教学, 既丰富了教学内容, 更体现了化 学与生活、社会、生产科技的紧密联系, 既提高学生学习元素及其化合物的热情, 更能激发学生的 好奇心, 激励学生热爱化学、学好化学, 培养学生运用化学知识解决实际问题的能力和创新思维。

\section{5 学会归纳总结}

本章的内容 “多、杂、散、乱”, 易学易忘。事实上, 本章内容并非完全杂乱无章, 也存在着 内在的联系和规律, 因此在学习过程中, 要指导学生及时复习教学内容, 引导学生学会分析教材内 容, 学会归纳知识及总结其内在规律。

如学完卤素的氢化物时, 要指导学生总结氟的氢化物具有特殊性性, $\mathrm{HF} \rightarrow \mathrm{Hl}$ 等性质递变规律 (见表4); 学完氯酸及其盐后, 要指导学生总结卤酸的氧化性具有反常性(见表4); 学完本章后要指导 学生对本族元素及其化合物的性质变化规律作总结(见表4)。

表4 卤族元素的相似性、递变性、特殊性、反常性

\begin{tabular}{|c|c|}
\hline 性质 & 内容 \\
\hline 相 & 1) 价电子构型为 $n s^{2} n p^{5}$, 最外电子层有7个电子 \\
\hline 似 & 2) 除氟外，正氧化态均有 $+1 、+3 、+5 、+7$ \\
\hline 性 & 3) 负氧化态均为 -1 \\
\hline & 4) 卤素单质(双原子分子)都有较强的氧化性，与金属、非金属、水、碱等反应 \\
\hline 递 & 1) 单质的颜色由浅到深, 状态由气态到液、固态, 熔、沸点由低到高, 密度由小到大, 水溶性由大到小 \\
\hline 变 & 2) 原子序数增大, 电子层数逐渐增大, 原子半径逐渐增大 \\
\hline 性 & 3) 单质的氧化性由强到弱; 与 $\mathrm{H}_{2} 、$ 金属、水等反应的程度由剧烈到微弱, 条件由易变难 \\
\hline & 4) 阴离子的还原性逐渐增强, 即 $\mathrm{F}^{-}<\mathrm{Cl}^{-}<\mathrm{Br}^{-}<\mathrm{I}^{-}$ \\
\hline & 5) 氢化物的稳定性逐渐减弱: $\mathrm{HF}>\mathrm{HCl}>\mathrm{HBr}>\mathrm{HI}$; 熔沸点逐渐增强: $\mathrm{HCl}<\mathrm{HBr}<\mathrm{HI}$ ( $\mathrm{HF}$ 除外); 还原性逐渐增强( $\mathrm{HF}$ 和 HCI可 \\
\hline & 用浓硫酸制取, $\mathrm{HBr}$ 和HI应用浓磷酸制取), 其水溶液(氢卤酸)的酸性逐渐增强 \\
\hline & 6) 同一元素不同氧化态的含氧酸, 高氧化态含氧酸的酸性较强, 低氧化态的含氧酸的酸性较弱, 如: $\mathrm{HClO}_{4}>\mathrm{HClO}_{3}>\mathrm{HClO}_{2}>\mathrm{HOCl}$ \\
\hline & 7) 同一族同种类型的含氧酸, 随电子层数增加酸性依次减弱, 如: $\mathrm{HClO}_{4}>\mathrm{HBrO}_{4}>\mathrm{H}_{5} \mathrm{IO}_{6}$ \\
\hline 特 & 1) 氟无正氧化态, 无含氧酸 \\
\hline 殊 & 2) 氟化银易溶于水, 且无感光性, 其他卤化银难溶于水 \\
\hline 性 & 3) 氟化钙难溶于水, 其他卤化钙易溶于水 \\
\hline & 4) $\mathrm{F}_{2}$ 跟水剧烈反应(除 $\mathrm{F}_{2}$ 外, 其他均可得到水溶液 “卤水”); $F_{2}$ 能跟稀有气体氙、氪等反应 \\
\hline & 5) 氢氟酸为弱酸, 但腐蚀性强, 能腐蚀玻璃 \\
\hline & 6) $\mathrm{Cl}_{2}$ 易液化; $\mathrm{Br}_{2}$ 是唯一的一种常温下呈液态的非金属单质且易挥发; $\mathrm{I}_{2}$ 易升华且能使淀粉溶液变蓝色 \\
\hline & 7) 高碘酸在酸性介质中能使 $\mathrm{Mn}^{2+}$ 氧化为 $\mathrm{MnO}_{4}^{-}: 2 \mathrm{Mn}^{2+}+5 \mathrm{H}_{5} \mathrm{IO}_{6}=2 \mathrm{MnO}_{4}^{-}+5 \mathrm{IO}_{3}^{-}+7 \mathrm{H}_{2} \mathrm{O}+11 \mathrm{H}^{+}$ \\
\hline 反 & 1) 氟化氢的熔、沸点反常升高 \\
\hline 常 & 2) 卤酸的氧化性: $\mathrm{HBrO}_{3}>\mathrm{HClO}_{3}>\mathrm{HIO}_{3}$ \\
\hline 性 & 3) 高卤酸的氧化性: $\mathrm{HBrO}_{4}>\mathrm{H}_{5} \mathrm{IO}_{6}>\mathrm{HClO}_{4}$ \\
\hline
\end{tabular}


归纳总结是整章学习过程最重要也是最后一个环节, 教师要培养学生善于归纳总结的良好习惯, 做到 “每天有复习, 每节有小结, 每章有总结”, 把知识编织成一张完整、系统的 “关系网”, 让学 生能及时查漏补缺, 扬长避短, 帮助学生把知识记得牢固、记得清晰, 从而提高学生学习效率, 从 本质上也提高课堂教学质量。

\section{3 结语}

元素化学历来是无机化学教学中的难点, 如何解决这一老大难问题, 一直是化学教育工作者不 断探索、追求的目标。本文以卤素课堂教学为例, 通过讲述卤素发现史润物无声地开展思政教育, 运用化学原理指导元素化学教学, 采用线上线下混合式模式教学, 结合实际应用讲透物质的性质, 指导学生学会归纳总结等方面开展课堂教学, 很好地把零散紊乱的知识梳理成有条理的知识结构, 帮助学生走出学习若境, 极大提高学生的学习积极性和主动性, 提升学生的自主学习能力, 从而提 高了课堂教学质量。

\section{参 考 文 献}

[1] 北京师范大学, 华中师范大学, 南京师范大学无机化学教研室. 无机化学(下册). 第4版. 北京: 高等教育出版社, 2003: 253-473.

[2] 魏明灯, 魏巧华. 大学化学, 2020, 35 (8), 24.

[3] 陈咏梅, 周云山, 王桂荣, 金銅. 大学化学, 2019, 34 (5), 15.

[4] 雷钰荣, 宁一诺, 朱亚先. 大学化学, 2018, 33 (7), 6 .

[5] 展鹏, 蒋小飞, 王艺铭, 兰共德, 刘新泳. 大学化学, 2019, 34 (11), 61.

[6] 朱国贤, 谢木标, 陈静, 潘荣楷. 大学化学, 2019, 34 (9), 50.

[7] 徐家宁, 史苏华, 宋天佑. 无机化学例题与习题. 北京: 高等教育出版社, 2000: 108-115. 